\title{
A construction of the Glashow-Weinberg-Salam model on the lattice with exact gauge invariance
}

\author{
Daisuke Kadoh* \\ Center for Computational Sciences, University of Tsukuba, Ibaraki 305-8571, Japan \\ E-mail:kadoh@ccs.tsukuba.ac.jp ${ }^{\dagger}$
}

\section{Yoshio Kikukawa}

Institute of Physics, University of Tokyo, Tokyo 153-8902, Japan

E-mail: kikukawa@hep1.c.u-tokyo.ac.jp

\begin{abstract}
We present a gauge-invariant and non-perturbative construction of the Glashow-Weinberg-Salam model on the lattice, based on the lattice Dirac operator satisfying the Ginsparg-Wilson relation. Our construction covers all SU(2) topological sectors with vanishing U(1) magnetic flux and would be usable for a description of the baryon number non-conservation. In infinite volume, it provides a gauge-invariant regularization of the electroweak theory to all orders of perturbation theory. First we formulate the reconstruction theorem which asserts that if there exists a set of local currents satisfying cetain properties, it is possible to reconstruct the fermion measure which depends smoothly on the gauge fields and fulfills the fundamental requirements such as locality, gauge-invariance and lattice symmetries. Then we give a closed formula of the local currents required for the reconstruction theorem.
\end{abstract}

The XXVI International Symposium on Lattice Field Theory

July 14-19 2008

Williamsburg, Virginia, USA

\footnotetext{
*Present address: Theoretical Physics Laboratory, RIKEN, Wako 2-1, Saitama 351-0198, Japan

$\dagger$ Present E-mail address: kadoh@ riken.jp

${ }^{\ddagger}$ Speaker.
} 


\section{The Glashow-Weinberg-Salam model on the lattice}

We describe a construction of the Glashow-Weinberg-Salam model on the lattice [1, 2] within the framework of chiral lattice gauge theories based on the lattice Dirac operator satisfying the Ginsparg-Wilson relation [3, 4]. We assume a local, gauge-covariant lattice Dirac operator $D$ which satisfies the Ginsparg-Wilson relation [5, 6, 7, 8, 9, 10, 11]. An explicit example of such lattice Dirac operator is given by the overlap Dirac operator [6, 9], which was derived from the overlap formalism [12, 13, 14, 15] / the domain wall fermion [16, 17, 18, 19, 20, 21]. With this choice, our formulation is equivalent to the overlap formalism for chiral lattice gauge theories, or the domain wall fermion approach [22, 23].

\subsection{SU(2) $\times \mathrm{U}(1)$ Gauge fields}

We consider the four-dimensional lattice of the finite size $L$ and choose lattice units,

$$
\Gamma=\left\{x=\left(x_{1}, x_{2}, x_{3}, x_{4}\right) \in \mathbb{Z}^{4} \mid 0 \leq x_{\mu}<L(\mu=1,2,3,4)\right\} .
$$

$\mathrm{SU}(2)$ and $\mathrm{U}(1)$ gauge fields on $\Gamma$ may be represented through periodic link fields $U^{(1)}(x, \mu) \in \mathrm{U}(1)$, $U^{(2)}(x, \mu) \in \mathrm{SU}(2)$ on the infinite lattice. We require the so-called admissibility condition on the plaquette variables $P^{(2)}(x, \mu, v), P^{(1)}(x, \mu, v)$ of gauge fields,

$$
\left|F_{\mu \nu}(x)\right|<\varepsilon_{1}, \quad\left\|1-P^{(2)}(x, \mu, v)\right\|<\varepsilon_{2} \quad(\text { for all } x, \mu, v),
$$

where $F_{\mu v}(x) \equiv \frac{1}{i} \ln P^{(1)}(x, \mu, v) \in(-\pi, \pi]$. This condition ensures that the overlap Dirac operator [6 9 is a smooth and local function of the gauge field if $Y \varepsilon_{1}+\varepsilon_{2}<1 / 30\left(\varepsilon_{1}, \varepsilon_{2}>0\right)$, where $Y$ is the hyper-charge of the fermion on which the overlap Dirac operator acts [11]. To impose the admissibility condition dynamically, we adopt the following action for the gauge fields:

$$
\begin{aligned}
S_{G}= & \frac{1}{g_{2}^{2}} \sum_{x \in \Gamma} \sum_{\mu, \nu} \operatorname{tr}\left\{1-P^{(2)}(x, \mu, \nu)\right\}\left[1-\operatorname{tr}\left\{1-P^{(2)}(x, \mu, \nu)\right\} / \varepsilon_{2}^{2}\right]^{-1} \\
& +\frac{1}{4 g_{1}^{2}} \sum_{x \in \Gamma \mu, \nu} \sum_{\mu \nu}\left[F_{\mu \nu}(x)\right]^{2}\left\{1-\left[F_{\mu \nu}(x)\right]^{2} / \varepsilon_{1}^{2}\right\}^{-1} .
\end{aligned}
$$

\subsection{Quarks and Leptons}

Right- and left- handed Weyl fermions are introduced on the lattice based on the GinspargWilson relation. Let us first consider a generic gauge group $G$ and a Dirac field $\psi(x)$ coupled to the gauge field $U(x, \mu)$ in a certain representaion $R$ of $G$. Given a local, gauge-covariant lattice Dirac operator $D_{L}$ which acts on $\psi(x)$ and satisfies the Ginsparg-Wilson relation, $\gamma_{5} D_{L}+D_{L} \gamma_{5}=$ $2 D_{L} \gamma_{5} D_{L}$, one can introduce a chiral operator as

$$
\hat{\gamma}_{5} \equiv \gamma_{5}\left(1-2 D_{L}\right), \quad\left(\hat{\gamma}_{5}\right)^{2}=\mathbb{I} .
$$

Then, the right- and left-handed Weyl fermions in the representaion $R$ of $G$ can be defined by the eigenstates of the chiral operator $\hat{\gamma}_{5}$ (and $\gamma_{5}$ for the anti-fields). Namely,

$$
\psi_{ \pm}(x)=\hat{P}_{ \pm} \psi(x), \quad \bar{\psi}_{ \pm}(x)=\bar{\psi}(x) P_{\mp}
$$


where $\hat{P}_{ \pm}$and $P_{ \pm}$are the chiral projection operators given by $\hat{P}_{ \pm}=\left(1 \pm \hat{\gamma}_{5}\right) / 2, P_{ \pm}=\left(1 \pm \gamma_{5}\right) / 2$.

Now we consider quarks and leptons in the Glashow-Weinberg-Salam model. For simplicity, we consider the first family. We adopt the convention for the normalization of the hyper-charges such that the Nishijima-Gell-Mann relation reads $Q=T_{3}+\frac{1}{6} Y$. To describe the left-handed quarks and leptons, which are $\mathrm{SU}(2)$ doublets, we introduce a left-handed fermion $\psi_{-}(x)$ with the index $\alpha(=1, \cdots, 4)$, each component of which couples to the $\mathrm{SU}(2) \times \mathrm{U}(1)$ gauge field, $U^{(2)}(x, \mu) \otimes$ $\left\{U^{(1)}(x, \mu)\right\}^{Y_{\alpha}}$, with the hyper-charge $Y_{\alpha}\left(Y_{1,2,3}=1\right.$ and $\left.Y_{4}=-3\right)$. Namely,

$$
\psi_{-}(x)={ }^{t}\left(q_{-}^{1}(x), q_{-}^{2}(x), q_{-}^{3}(x), l_{-}(x)\right) .
$$

Similarly, to describe the right-handed quarks and leptons, which are SU(2) singlets, we introduce a right-handed fermion $\psi_{+}(x)$ with the index $\beta(=1, \cdots, 8)$, each component of which couples to the $\mathrm{U}(1)$ gauge field, $\left\{U^{(1)}(x, \mu)\right\}^{Y_{\beta}}$, with the hyper-charge $Y_{\beta}\left(Y_{1,3,5}=4, Y_{2,4,6}=-2, Y_{7}=0\right.$ and $\left.Y_{8}=-6\right)$. Namely,

$$
\psi_{+}(x)={ }^{t}\left(u_{+}^{1}(x), d_{+}^{1}(x), u_{+}^{2}(x), d_{+}^{2}(x), u_{+}^{3}(x), d_{+}^{3}(x), v_{+}(x), e_{+}(x)\right) .
$$

Then the action of quarks and leptons is given by

$$
S_{F}=\sum_{x \in \Gamma} \bar{\psi}_{-}(x) D_{L} \psi_{-}(x)+\sum_{x \in \Gamma} \bar{\psi}_{+}(x) D_{L} \psi_{+}(x) .
$$

\subsection{Higgs field and its Yukawa-couplings to quarks and leptons}

Higgs field is a SU(2) doublet with the hyper-charge $Y_{h}=+3$. The action of the Higgs field may be given by

$$
S_{H}=\sum_{x}\left[\sum_{v}\left(\nabla_{v} \phi(x)\right)^{\dagger} \nabla_{v} \phi(x)+\frac{\lambda}{2}\left(\phi(x)^{\dagger} \phi(x)-v^{2}\right)^{2}\right],
$$

where $\phi(x)$ couples to the gauge field $U^{(2)}(x, \mu) \otimes\left\{U^{(1)}(x, \mu)\right\}^{Y_{h}}$ and $\nabla_{v}$ is the $\mathrm{SU}(2) \times \mathrm{U}(1)$ gauge-covariant difference operator. Yukawa couplings of the Higgs field to the quarks and leptons may also be introduced as follows ${ }^{1}$ :

$$
\begin{array}{r}
S_{Y}=\sum_{x}\left[y_{u} \bar{q}_{-}^{i}(x) \tilde{\phi}(x) u_{+}^{i}(x)+y_{u}^{*} \bar{u}_{+}^{i}(x) \tilde{\phi}(x)^{\dagger} q_{-}^{i}(x)\right. \\
+y_{d} \bar{q}_{-}^{i}(x) \phi(x) d_{+}^{i}(x)+y_{d}^{*} \bar{d}_{+}^{i}(x) \phi(x)^{\dagger} q_{-}^{i}(x) \\
\left.+y_{l} \bar{l}_{-}(x) \phi(x) e_{+}(x)+y_{l}^{*} \bar{e}_{+}(x) \phi(x)^{\dagger} l_{-}(x)\right]
\end{array}
$$

where $\tilde{\phi}(x)$ is the $\mathrm{SU}(2)$ conjugate of $\phi(x)$.

Thus the total lattice action, $S=S_{G}+S_{F}+S_{H}+S_{Y}$, defines a classical theory of the GlashowWeinberg-Salam model on the lattice with the first-family quarks and leptons. In this action, locality, gauge-invariance and lattice symmetries such as translations and rotations are manifest. CP symmetry, however, is not manifest even with the real Yukawa couplings. But it is possible to show that at the quantum level both the partition function and the on-shell amplitudes respect the $\mathrm{CP}$ symmetry [24, 25]. With the three families, then, the breaking of CP symmetry comes from the Kobayashi-Maskawa phase [26] as in the continuum theory.

\footnotetext{
${ }^{1}$ One may add the Dirac-type mass term for the neutrino, $\sum_{x}\left\{y_{v} \bar{l}_{-}(x) \tilde{\phi}(x) v_{+}(x)+y_{v}^{*} \bar{v}_{+}(x) \tilde{\phi}(x)^{\dagger} l_{-}(x)\right\}$.
} 


\subsection{Topology of the $S U(2) \times U(1)$ gauge fields}

The admissibility condition allows us to define a topological charge of the gauge fields through the lattice Dirac operator $D_{L}$ [12, 13, 8, 10]: for the admissible $\mathrm{SU}(2)$ and U(1) gauge fields, one has

$$
Q^{(i)}=\left.\sum_{x \in \Gamma} \operatorname{tr}\left\{\gamma_{5}\left(1-D_{L}\right)\right\}(x, x)\right|_{U=U^{(i)}} \quad(i=1,2) .
$$

For $0<\varepsilon_{1}<\pi / 3$, the admissible $\mathrm{U}(1)$ gauge fields can also be classified by the magnetic fluxes,

$$
m_{\mu \nu}=\frac{1}{2 \pi} \sum_{s, t=0}^{L-1} F_{\mu \nu}(x+s \hat{\mu}+t \hat{v}),
$$

which are integers independent of $x$. $m_{\mu \nu}$ is related to $Q^{(1)}$ by $Q^{(1)}=(1 / 2) \sum_{\mu \nu} m_{\mu \nu}^{2}$ [27]. Then the admissible $\mathrm{SU}(2)$ and $\mathrm{U}(1)$ gauge fields may be classified by the topological numbers $Q_{2}$ and $m_{\mu \nu}$, respectively. We denote the space of the admissible $\mathrm{SU}(2)$ gauge fields with a given topological charge $Q^{(2)}$ by $\mathfrak{U}^{(2)}[Q]$ and the space of the admissible $\mathrm{U}(1)$ gauge fields with a given magnetic fluxes $m_{\mu \nu}$ by $\mathfrak{U}^{(1)}[\mathrm{m}]$. Strictly speaking, the complete topological classification of the space of admissible SU(2) gauge fields is not known yet. But, as we will see, our construction is valid for any $\mathrm{SU}(2)$ topological sectors as long as the $\mathrm{U}(1)$ magnetic flux vanishes identically.

\section{Reconstruction theorem of the fermion measure}

The properties of the fermion measure can be characterized by the so-called measure term [3, 4] which is given in terms of the chiral basis and its variation with respect to the gauge fields as

$$
\mathfrak{L}_{\eta}=i \sum_{j}\left(v_{j}, \delta_{\eta} v_{j}\right)+i \sum_{j}\left(u_{j}, \delta_{\eta} u_{j}\right) .
$$

Similar to the case of U(1) chiral lattice gauge theories [3], one can establish the following theorem in the lattice Glashow-Weinberg-Salam model.

Theorem: In the topological sectors with vanishing $\mathrm{U}(1)$ magnetic flux, $\mathfrak{U}^{(2)}[Q] \otimes \mathfrak{U}^{(1)}[0]$, if there exist local currents $j_{\mu}^{a}(x)(a=1,2,3), j_{\mu}(x)$ which satisfy the following four plus one properties, it is possible to reconstruct the fermion measure (the bases $\left\{u_{j}(x)\right\},\left\{v_{j}(x)\right\}$ ) which depends smoothly on the gauge fields and fulfills the fundamental requirements such as locality, gauge-invariance, integrability and lattice symmetries:

1. $j_{\mu}^{a}(x), j_{\mu}(x)$ are defined for all admissible $\mathrm{SU}(2) \times \mathrm{U}(1)$ gauge fields in the given topological sectors and depends smoothly on the link variables.

2. $j_{\mu}^{a}(x)$ and $j_{\mu}(x)$ are gauge-covariant and -invariant, respectively and both transform as axial vector currents under the lattice symmetries.

3. The linear functional $\mathfrak{L}_{\eta}=\sum_{x \in \Gamma}\left\{\eta_{\mu}^{a}(x) j_{\mu}^{a}(x)+\eta_{\mu}(x) j_{\mu}(x)\right\}$ is a solution of the integrability condition

$$
\delta_{\eta} \mathfrak{L}_{\zeta}-\delta_{\zeta} \mathfrak{L}_{\eta}+\mathfrak{L}_{[\eta, \zeta]}=i \operatorname{Tr}\left\{\hat{P}_{-}\left[\delta_{\eta} \hat{P}_{-}, \delta_{\zeta} \hat{P}_{-}\right]\right\}+i \operatorname{Tr}\left\{\hat{P}_{+}\left[\delta_{\eta} \hat{P}_{+}, \delta_{\zeta} \hat{P}_{+}\right]\right\}
$$

for all periodic variations $\eta_{\mu}^{a}(x), \eta_{\mu}(x)$ and $\zeta_{\mu}^{a}(x), \zeta_{\mu}(x)$. 
4. The anomalous conservation laws hold:

$$
\begin{aligned}
\left\{\nabla_{\mu}^{*} j_{\mu}\right\}^{a}(x) & =\operatorname{tr}\left\{T^{a} \gamma_{5}(1-D)(x, x)\right\} \\
\partial_{\mu}^{*} j_{\mu}(x) & =\operatorname{tr}\left\{Y_{-} \gamma_{5}\left(1-D_{L}\right)(x, x)\right\}-\operatorname{tr}\left\{Y_{+} \gamma_{5}\left(1-D_{L}\right)(x, x)\right\}
\end{aligned}
$$

where $Y_{-}=\operatorname{diag}(1,1,1,-3)$ and $Y_{+}=\operatorname{diag}(4,-2, \cdots, 0,-6)$.

5. The current $j_{\mu}(x)$ has the scaling property with respect to the hyper-charge:

$$
j_{\mu}(x)=\sum_{\alpha=1}^{4} Y_{\alpha} F_{\mu-}\left[x ;\left\{U^{(1)}(x, \mu)\right\}^{Y_{\alpha}}, U^{(2)}(x, \mu)\right]+\sum_{\beta=1}^{8} Y_{\beta} F_{\mu+}\left[x ;\left\{U^{(1)}(x, \mu)\right\}^{Y_{\beta}}\right],
$$

where $F_{ \pm}$are certain local functionals of the link variables.

\section{A sketch of the proof:}

The smooth fermion measure exists if and only if the integrability condition holds true for any closed curve $U_{t}\left(t \in[0,1] ; U_{1}=U_{0}\right)$ in the space of the admissible gauge fields:

$$
W=\operatorname{det}\left(1-P_{0-}+P_{0-} Q_{1-}\right) \operatorname{det}\left(1-P_{0+}+P_{0+} Q_{1+}\right),
$$

where $W$ is defined by

$$
W \equiv \exp \left\{i \int_{0}^{1} d t \mathfrak{L}_{\eta}\right\}, \quad \eta_{\mu}(x)=i \partial_{t} U_{t}(x, \mu) U_{t}(x, \mu)^{-1}
$$

and $Q_{ \pm t}$ is defined by the evolution operator of the projector $P_{t \pm}=\left.\hat{P}_{ \pm}\right|_{U=U_{t}}$ satisfying

$$
\partial_{t} Q_{t \pm}=\left[\partial_{t} P_{t \pm}, P_{t \pm}\right] Q_{t \pm}, \quad Q_{0 \pm}=1
$$

Since $\mathfrak{U}^{(2)}[Q] \otimes \mathfrak{U}^{(1)}[0]$ is a direct product space, any non-contractible loop in $\mathfrak{U}^{(2)}[Q] \otimes \mathfrak{U}^{(1)}[0]$ may be deformed to the product of the loops in $\mathfrak{U}^{(2)}[Q]$ and $\mathfrak{U}^{(1)}[0]$, respectively. Then, in order to prove the global integrability condition, one may consider separately the following two cases, (1) noncontractible loops in $\mathfrak{U}^{(2)}[Q]$ with the trivial U(1) link field as a background and (2) non-contactible loops in $\mathfrak{U}^{(1)}[0]$ with an arbitrarily chosen $S U(2)$ link field in $\mathfrak{U}^{(2)}[Q]$ as a background.

The classification of the non-contractible loops in $\mathfrak{U}^{(2)}[Q]$ is not known so far [28]. When $U^{(1)}(x, \mu)=1$, however, the left-handed fermion $\psi_{-}(x)={ }^{t}\left(q_{-}^{1}(x), q_{-}^{2}(x), q_{-}^{3}(x), l_{-}(x)\right)$ consists of four degenerate $\mathrm{SU}(2)$ doublets and one may choose the basis vectors of $\psi_{-}(x)$ for any given $\mathrm{SU}(2)$ gauge field $U^{(2)}(x, \mu) \in \mathfrak{U}^{(2)}[Q]$ as follows:

$$
\begin{aligned}
q_{-}^{1}(x) & =\sum_{j} w_{j}(x) c_{j}^{1}, \\
q_{-}^{2}(x) & =\sum_{j}\left(\gamma_{5} C^{-1} \otimes i \sigma_{2}\right)\left[w_{j}(x)\right]^{*} c_{j}^{2}, \\
q_{-}^{3}(x) & =\sum_{j} w_{j}(x) c_{j}^{3}, \\
l_{-}(x) & =\sum_{j}\left(\gamma_{5} C^{-1} \otimes i \sigma_{2}\right)\left[w_{j}(x)\right]^{*} c_{j}^{4},
\end{aligned}
$$


where $\left\{w_{j}(x)\right\}$ is an arbitrarily chosen basis for a single left-handed SU(2) doublet. With this choice of the basis, one can infer that the measure term vanishes identically and therefore $W=1$. On the other hand, from the charge conjugation property of $Q_{t-}$,

$$
Q_{t-}=\oplus_{i=1}^{4} Q_{t-(i)}, \quad Q_{t-(i)}=\left(\gamma_{5} C^{-1} \otimes i \sigma_{2}\right)\left\{Q_{t-(i)}^{-1}\right\}^{T}\left(C \gamma_{5} \otimes\left(i \sigma_{2}\right)^{-1}\right)
$$

it follows that

$$
\operatorname{det}\left(1-P_{0-}+P_{0-} Q_{1-}\right)=\left[\operatorname{det}\left(1-P_{0-(1)}+P_{0-(1)} Q_{1-(1)}\right)\right]^{4}=1 .
$$

Thus the measure of the chiral fermion $\psi_{-}(x)$ can be defined globally within $\mathfrak{U}^{(2)}[Q]$ and the lattice counterpart of the SU(2) global anomaly [29, 30, 31] is absent in this case.

The non-trivial U(1) loops consist of the loops along the gauge orbits (gauge loops) and the loops along the Wilson lines (non-gauge loops), as shown in [3]. For gauge loops, the proof goes just like the case of the abelian theories [3]. For non-gauge loops, one has

$$
\mathfrak{L}_{\eta}=\left.2 \pi j_{v}(0)\right|_{U^{(2)} \otimes U_{t}^{(1)}}, \quad \eta_{\mu}(x)_{(v)}=-i U_{[w]}(x, \mu)^{-1} \partial_{t_{v}} U_{[w]}(x, \mu)=2 \pi \delta_{\mu v} \delta_{\tilde{x} 0}
$$

where the $\mathrm{SU}(2)$ gauge field $U^{(2)}(x, \mu)$ is chosen arbitrarily in $\mathfrak{U}^{(2)}[Q]$ and is fixed as a background. Noting the scaling property of the U(1) measure term current, the l.h.s. can be evaluated as

$$
W=\exp \left\{i\left(\sum_{\alpha=1}^{4} Y_{\alpha}\right) \int_{0}^{2 \pi} d t F_{v-}(0)\right\} \cdot \exp \left\{i\left(\sum_{\beta=1}^{8} Y_{\beta}\right) \int_{0}^{2 \pi} d t F_{v+}(0)\right\}=1 .
$$

On the other hand, from the factorization properties of $Q_{1 \pm}$,

$$
Q_{1-}=\oplus_{\alpha=1}^{4}\left\{\left.Q_{1-}\right|_{Y=1}\right\}^{Y_{\alpha}}, \quad Q_{1+}=\oplus_{\beta=1}^{8}\left\{\left.Q_{1+}\right|_{Y=1}\right\}^{Y_{\beta}}
$$

the r.h.s. can be evaluated as

$$
\begin{aligned}
& \operatorname{det}\left\{1-P_{0 \pm}+P_{0 \pm} Q_{1 \pm}\right\} \\
& =\left(\left.\operatorname{det}\left\{1-P_{0 \pm}+P_{0 \pm} Q_{1 \pm}\right\}\right|_{Y=1}\right)^{\operatorname{tr}\left\{Y_{ \pm}\right\}}=1
\end{aligned}
$$

This completes the proof of the global integrability condition, and therefore, the smoothness of the fermion measure. Locality, gauge-invarinace and lattice symmetries of the fermion measure also follow from the properties of the measure term currents, $j_{\mu}^{a}(x)(a=1,2,3), j_{\mu}(x) \llbracket 1$.

\section{An explicit construction of the mesure term}

It is indeed possible to construct the local currents $j_{\mu}^{a}(x)(a=1,2,3)$ and $j_{\mu}(x)$ which satisfy all the required properties for the reconstruction theorem. Full details of our construction is given in [1], which is an extension of the construction of $U(1)$ chiral gauge theories [3, 32, 33] to the case of the $\mathrm{SU}(2) \times \mathrm{U}(1)$ chiral gauge theory. 


\section{References}

[1] D. Kadoh and Y. Kikukawa, JHEP 0805, 095 (2008) [arXiv:0709.3658 [hep-lat]].

[2] Y. Kikukawa and Y. Nakayama, Nucl. Phys. B 597, 519 (2001) [arXiv:hep-lat/0005015].

[3] M. Lüscher, Nucl. Phys. B 549, 295 (1999) [arXiv:hep-lat/9811032].

[4] M. Lüscher, Nucl. Phys. B 568, 162 (2000) [arXiv:hep-lat/9904009].

[5] P. H. Ginsparg and K. G. Wilson, Phys. Rev. D 25, 2649 (1982).

[6] H. Neuberger, Phys. Lett. B 417, 141 (1998) [arXiv:hep-lat/9707022].

[7] P. Hasenfratz, Nucl. Phys. B 525, 401 (1998) [arXiv:hep-lat/9802007].

[8] P. Hasenfratz, V. Laliena and F. Niedermayer, Phys. Lett. B 427, 125 (1998) [arXiv:hep-lat/9801021].

[9] H. Neuberger, Phys. Lett. B 427, 353 (1998) [arXiv:hep-lat/9801031].

[10] M. Lüscher, Phys. Lett. B 428, 342 (1998) [arXiv:hep-lat/9802011].

[11] P. Hernandez, K. Jansen and M. Lüscher, Nucl. Phys. B 552, 363 (1999) [arXiv:hep-lat/9808010].

[12] R. Narayanan and H. Neuberger, Nucl. Phys. B 412, 574 (1994) [arXiv:hep-lat/9307006].

[13] R. Narayanan and H. Neuberger, Phys. Rev. Lett. 71, 3251 (1993) [arXiv:hep-lat/9308011].

[14] R. Narayanan and H. Neuberger, Nucl. Phys. B 443, 305 (1995) [arXiv:hep-th/9411108].

[15] Y. Kikukawa and H. Neuberger, Nucl. Phys. B 513, 735 (1998) [arXiv:hep-lat/9707016].

[16] D. B. Kaplan, Phys. Lett. B 288, 342 (1992) [arXiv:hep-lat/9206013].

[17] Y. Shamir, Nucl. Phys. B 406, 90 (1993) [arXiv:hep-lat/9303005].

[18] V. Furman and Y. Shamir, Nucl. Phys. B 439, 54 (1995) [arXiv:hep-lat/9405004].

[19] P. M. Vranas, Phys. Rev. D 57, 1415 (1998) [arXiv:hep-lat/9705023].

[20] H. Neuberger, Phys. Rev. D 57, 5417 (1998) [arXiv:hep-lat/9710089].

[21] Y. Kikukawa and T. Noguchi, arXiv:hep-lat/9902022.

[22] Y. Kikukawa, Phys. Rev. D 65, 074504 (2002) [arXiv:hep-lat/0105032].

[23] T. Aoyama and Y. Kikukawa, arXiv:hep-lat/9905003.

[24] K. Fujikawa, M. Ishibashi and H. Suzuki, JHEP 0204, 046 (2002) [arXiv:hep-lat/0203016].

[25] L. Giusti, Nucl. Phys. Proc. Suppl. 119, 149 (2003) [arXiv:hep-lat/0211009].

[26] M. Kobayashi and T. Maskawa, Prog. Theor. Phys. 49, 652 (1973).

[27] T. Fujiwara, H. Suzuki and K. Wu, Phys. Lett. B 463, 63 (1999) [arXiv:hep-lat/9906016].

[28] D. H. Adams, Nucl. Phys. B 640, 435 (2002) [arXiv:hep-lat/0203014].

[29] H. Neuberger, Phys. Lett. B 437, 117 (1998) [arXiv:hep-lat/9805027].

[30] O. Bar, Nucl. Phys. B 650, 522 (2003) [arXiv:hep-lat/0209098].

[31] O. Bar and I. Campos, Nucl. Phys. B 581, 499 (2000) [arXiv:hep-lat/0001025].

[32] D. Kadoh, Y. Kikukawa and Y. Nakayama, JHEP 0412, 006 (2004) [arXiv:hep-lat/0309022].

[33] D. Kadoh and Y. Kikukawa, JHEP 0802, 063 (2008) [arXiv:0709.3656 [hep-lat]]. 